\title{
Connecting in-situ X-ray Nano- and Micro-Tomography via Fluid Dynamics Simulations to Study Barium Sulfate Mineral Precipitation
}

Vitalii Starchenko*1, Ke Yuan ${ }^{1}$, Juliane Weber $^{2}$, Michael Cheshire $^{1}$, Nikhil Rampal ${ }^{1}$, Andrew Stack $^{1}$

${ }^{1}$ Chemical Sciences Division, Oak Ridge National Laboratory, 1 Bethel Valley Rd, Oak Ridge, TN 37830 (*correspondence: starchenkov@ornl.gov)

2 The University of Arizona, Tucson, AZ 85721

It has been a great challenge for geochemical community to capture and distinguish the most important factors that govern mineral precipitation in porous materials and to couple physical and chemical processes happening on length and time scales that differ by several orders of magnitude.

Here, we present results on combining two sets of in-situ X-ray computed tomography (XCT) experiments (nano-XCT: $30 \mathrm{~nm}$ per voxel and micro-XCT: $1240 \mathrm{~nm}$ per voxel) with fluid dynamics simulations (CFD) that allows us to unravel coupling between reactant transport and local mineral growth in porous matrices. We used the supersaturated solutions containing barium $\left(\mathrm{Ba}^{2+}\right)$ and sulfate $\left(\mathrm{SO}_{4}^{2-}\right)$ ions that form a sparingly soluble salt - barite $\left(\mathrm{BaSO}_{4}\right)$. We have been able to show that quantitative analysis of micro-XCT images becomes possible, such as, the total amount of precipitated mineral and cluster analysis. The results confirm a strong correlation between transport efficiency and amount of precipitated mineral. In conjunction, the information on crystal nucleation process was obtained from nano-XCT. Additionally, strontium $\left(\mathrm{Sr}^{2+}\right)$ ions were used to model impurity presence in solution. We observed that the addition of strontium decreased the growth rate and increased the nucleation rate during precipitation process, indicating that the impurity has opposite effects on the crystal nucleation and crystal growth processes. 